OPEN

SUBJECT AREAS:

IMAGING

NEUROSCIENCE

COMPUTATIONAL

NEUROSCIENCE

MODELLING

Received

24 May 2012

Accepted

21 September 2012

Published

15 October 2012

Correspondence and requests for materials should be addressed to

P.F.A. (pablo. argibay@

hospitalitaliano.org.

ar)

The computational influence of

\title{
neurogenesis in the processing of spatial information in the dentate gyrus
}

\author{
Javier I. Cuneo, Nicolas H. Quiroz, Victoria I. Weisz \& Pablo F. Argibay
}

Laboratory for Biological \& Artificial Learning, Instituto de Ciencias Básicas y Medicina Experimental, Hospital Italiano de Buenos Aires, Potosi 4240 (1 199), Buenos Aires, Argentina.

This study was designed to analyze the effect of hippocampal neurogenesis on the spatial maps of granule cells. Accordingly, we developed and improved an artificial neural network that was originally proposed by Aimone. Many biological processes were included in this revised model to improve the biological relevance of the results. We proposed a novel learning-testing protocol to analyze the activation of encoding place cells across contexts and over time in the dentate gyrus. We observed that, regardless of the presence of neurogenesis, the quantity and morphology of the place fields were represented in the same manner by granule cells. Additionally, we observed that neurogenesis was an effective mechanism for reducing the degree of rate remapping that occurred in the place fields of the granule cells.

T he function of neurogenesis has been widely examined. However, despite the existence of many theoretical and practical studies, none have provided a clear explanation regarding the purpose of neurogenesis ${ }^{1}$. Here, we used a modified version of the computational model that was proposed by Aimone et al. in $2009^{2}$. Using this modified model and analyzing the manner in which the representation of new environments changes over time, we designed a novel model of learning in which the capacity of the dentate gyrus to learn and to retain new information (represented by spatial environments with olfactory stimuli) is evaluated. This new approach, in which both the place maps and neurogenesis are examined, connects two aspects of hippocampal function that have not previously been examined together. With respect to this study, two concepts that are related to the spatial activities of the hippocampus and may be influenced by neurogenesis.

The first concept is that of place fields, which are associated with the neurocomputation of spatial information in the hippocampus ${ }^{3-6}$. Place fields (PFs) have been experimentally supported and are specific locations in which place cells fire when a rat moves through a particular location in space. Granule cells (GCs) of the dentate gyrus (DG) typically exhibit multiple PFs, whereas CA3 cells have only a single $\mathrm{PF}^{5}$. It has been hypothesized that PF emergence begins with entorhinal grid cell neuronal activity ${ }^{8-10}$.

When activity of the DG and the PF were correlated in a model of the CA3 region, the CA3 processes that produced a single PF did not require direct cortical inputs to CA3 but depended critically on the competitive network processes that were implemented in the $\mathrm{DG}^{11}$. Therefore, a two-step mechanism that involves inputs to the DG from the entorhinal cortex (EC) and competitive processing in the DG can explain how grid cell inputs to the hippocampus produce cells that ultimately exhibit spatial selectivity.

The second concept is that of remapping. Remapping refers to the formation of distinct representations in populations of place cells following minor alterations in the inputs to the hippocampus ${ }^{12}$. Remapping displays two different modes: global remapping and rate remapping ${ }^{6}$. Global remapping consists of a complete reorganization of the "place code" as expressed through independent rate and place distributions in different test environments ${ }^{13}$. Rate remapping refers to a selective alteration in firing rate distributions but does not include an altered "place code" ${ }^{\prime 5,7,13,14}$. A mechanism that can be used for DG rate remapping was recently proposed. Through computational methods, it was inferred that the convergence of information from the lateral EC (IEC) and the medial EC $(\mathrm{mEC})$, in conjunction with a feedback inhibition-mediated competitive network process, can quantitatively account for rate remapping ${ }^{10}$.

The objective of the present study was to determine whether the spatial maps of the hippocampus are continuously expressed in the same manner in the presence or absence of neurogenesis and the method by which neurogenesis influences rate remapping using a plausible model of hippocampal neurogenesis. 


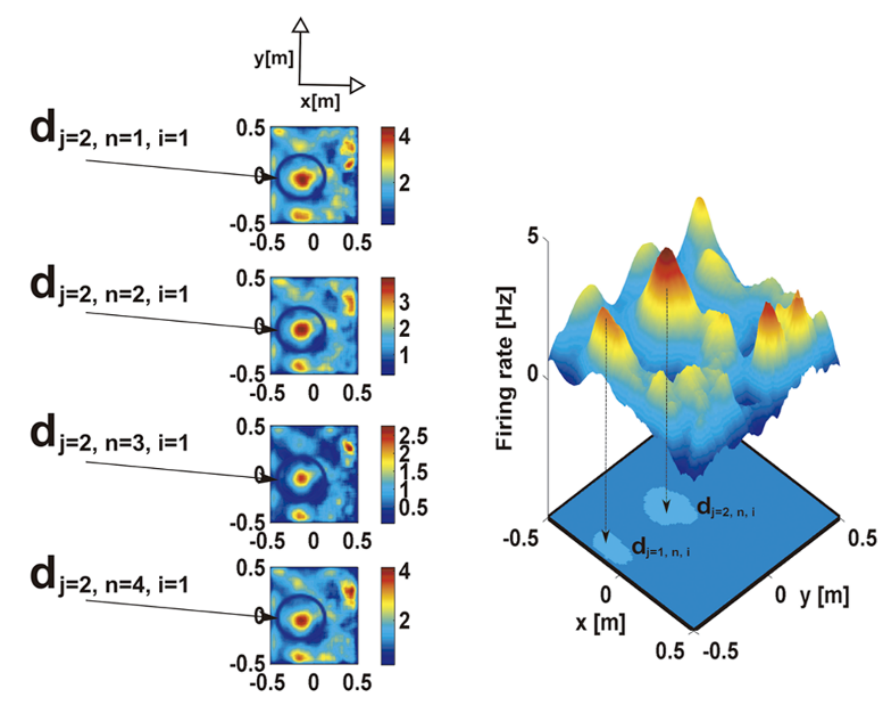

Figure 1 Representation of the detection and the analysis of place fields. The peak of the PF was designated as the coefficient $\mathbf{d}$. The suffix $\mathbf{i}$ represents the environment being studied, the suffix $\mathbf{n}$ represents the retrieval of the environment, and the suffix $\mathbf{j}$ represents the number of detected PFs. In these examples, $\mathbf{i}$ was held constant, meaning that only one environment was being studied; $\mathbf{n}=1$ indicates the first test of the first environment, and the suffix $\mathbf{n}$ represents the retrieval number of this environment after learning a novel environment.

\section{Results}

Dentate gyrus without neurogenesis. To determine the influence of neurogenesis on spatial information and the mechanisms by which new memories affect the representation of the oldest memories, we simulated two artificial neural networks. These networks were designed to compare the activity of a DG in which neurogenesis was inhibited and a DG in which neurogenesis was occurring.

A full analysis of the network lacking neurogenesis was performed following the training phase. First, the neurons that encoded the environment were identified by calculating the firing rate of each neuron in the layer. The neurons that exhibited a firing rate that surpassed a threshold of $1 \mathrm{~Hz}$ were considered to be the encoding neurons (see Supplementary Fig. S10).

Among a total of 800 mature GCs, only $7.26 \% \pm 4.82$ (mean \pm s.d., $\mathrm{N}=7$ ) encoded the environment. The mean firing rate of the environment-encoding cells was $1.98 \mathrm{~Hz} \pm 0.84$ (mean \pm s.d., $\mathrm{N}=7$ ), and the mean firing rate of the cells that did not encode an environment was $0.24 \mathrm{~Hz} \pm 0.25$ (mean \pm s.d., $\mathrm{N}=7$ ).

Based on the list of neurons that encoded each environment, the spatial representation of each cell over time was plotted to perform quantitative and qualitative analyses. The first qualitative result was obtained when we analyzed the PFs of the environment-encoding neurons; we observed that these PFs were approximately constant throughout the new learned environments (see Fig. 1).

After identifying the cells that encoded the environment, we quantified the number of detected PFs. We observed that each encoding cell encoded 2.13 place fields \pm 0.89 (mean \pm s.d., $\mathrm{N}=7$ ).

Because the objective of the present study was to analyze the effect of neurogenesis on the spatial representation of GCs, we analyzed rate remapping in the encoding cells PFs. First, to compare the results, we simulated a network in which neurogenesis was eliminated, and we sought to determine the rate remapping coefficients for all of the PFs over all of the retrievals as explained in the Methods section. Because the coefficients of rate remapping followed a normal distribution, a one-way ANOVA test was performed for the retrieval comparisons that were obtained using equation (1.1); no significant differences were detected in this analysis $\left(\mathrm{F}_{5,348}=1.76, \mathrm{P}>0.05\right)$. These results indicate that the change in the PFs with every new learned environment exhibited a similar mean rate remapping coefficient. Based on equation (1.2), the mean rate remapping coefficient of each retrieval comparison was calculated to quantify the change in the PF in a network lacking neurogenesis (see equation (1.3)). We next plotted the mean rate remapping coefficients of the retrieval comparisons (see Fig. 2). A mean rate remapping coefficient of $27.23 \% \pm 20.12$ (mean \pm s.d. pool, $N=6$ ) was determined for the PFs in the network lacking neurogenesis.

Dentate gyrus with neurogenesis. The encoding cells were then identified in a neural network in which neurogenesis was incorporated (see

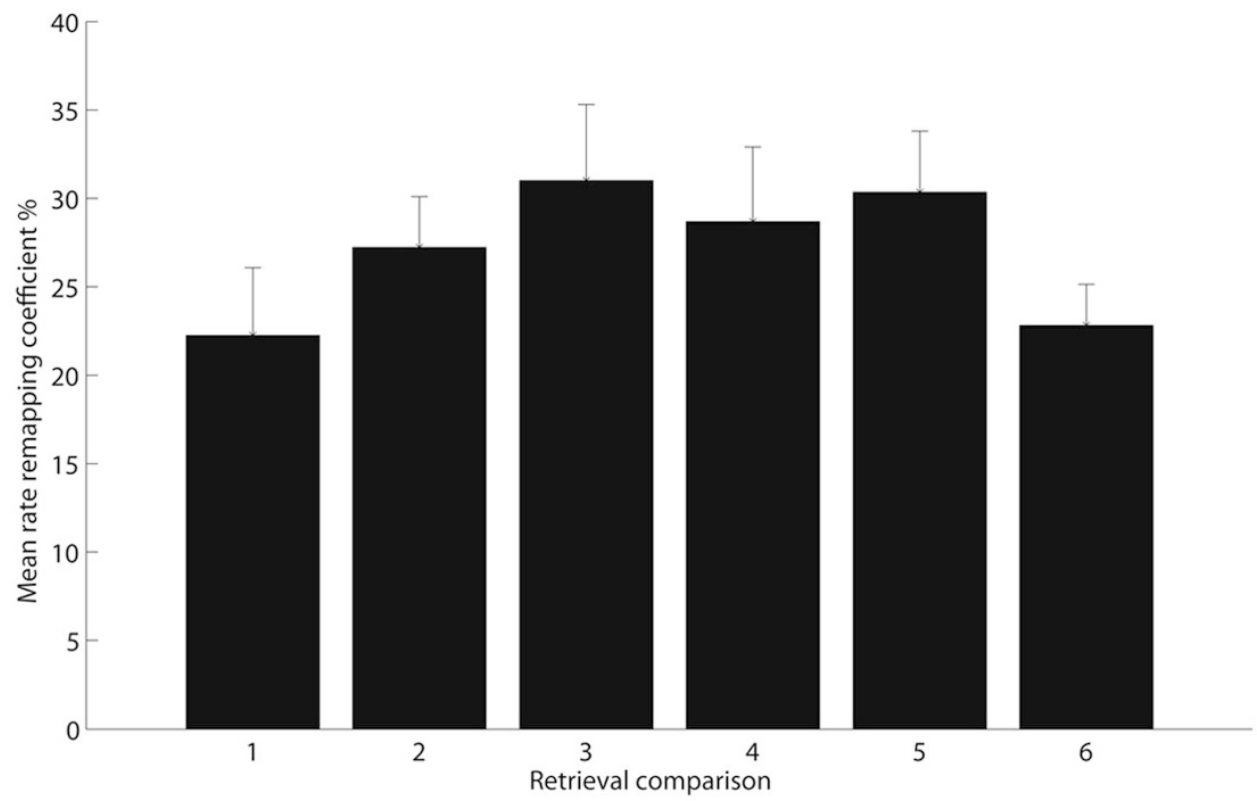

Figure $2 \mid$ Mean rate remapping coefficient in a network without neurogenesis. The mean rate remapping coefficient of the retrieval comparisons (mean \pm s.e.m.). The mean rate remapping of the columns was $27.23 \% \pm 20.12$ (mean \pm s.d. pool,$N=6$ ). In a network in which neurogenesis was suppressed, the magnitude of the change in the PFs of each cell showed no statistically significant difference. 
Supplementary Fig. S11). We observed that $8.33 \% \pm 4.57$ (mean \pm s.d., $\mathrm{N}=7$ ) of the GC layer encoded the environment. The environment-encoding cells exhibited a firing rate of $2.43 \mathrm{~Hz} \pm 1.28$ (mean \pm s.d., $\mathrm{N}=7$ ), whereas the cells that did not encode the environment exhibited a firing rate of $0.14 \mathrm{~Hz} \pm 0.22$ (mean \pm s.d., $\mathrm{N}=7$ ).

Using the new list of encoding neurons, we applied the PF detection algorithm to the place maps of these cells. We observed a mean of 2.37 place fields \pm 1.07 (mean \pm s.d., $N=7$ ) per encoding cell. Supplementary Fig. S12 reveals that the PFs of an adult neurogenic neuron remain constant, indicating that new neurons behave similarly to older neurons.

Similar to the simulation without neurogenesis, we analyzed the PFs of the encoding cells to identify the rate remapping coefficients in the neurogenesis model. A one-way ANOVA test was performed between the retrieval comparisons. This analysis indicated the presence of no significant differences between them $\left(\mathrm{F}_{2,93}=0.71, \mathrm{P}>\right.$ $0.05)$. The mean rate remapping coefficients of the retrieval comparisons were calculated, as shown in Fig. 3. The mean rate remapping of the network with neurogenesis was $14.25 \% \pm 10.58$ (mean \pm s.d. pool, $\mathrm{N}=6$ ). Considering that the mean coefficient of rate remapping of a network without neurogenesis was $27.23 \% \pm 20.12$ (mean \pm s.d. pool, $\mathrm{N}=6$ ), it could be concluded that neurogenesis decreases the interference of new environments with the previously encoded environments (see Fig. 2). Furthermore, as previously mentioned, new environments were preferentially encoded by new adult neurons.

Analysis of areas. The areas of the PFs of the GCs were measured to fully examine the effect of neurogenesis on spatial encoding in the DG. The area of each PF was obtained via the segmentation of the PFs; this step was followed by binarization. Histograms of the areas of the PFs of a DG with neurogenesis and of a DG lacking neurogenesis are shown in Fig. 4. None of the modeled DGs exhibited normally distributed PF areas; therefore, we performed a Mann-Whitney U Test, which revealed no differences between the medians of the PF areas of the different models $(\mathrm{Z}=-0.58, \mathrm{P}>0.05)$.

\section{Discussion}

Several simulations using neural networks have been previously developed to explain the computational implication of neurogenesis in memory-related processes (reviewed in Aimone et al. $2011^{15}$ ). However, we believe that the importance of neurogenesis in spatial information coding was not fully considered in these models. Therefore, we performed a biologically plausible simulation to compare a network with a static architecture (without neurogenesis) and a dynamic network in which additive neurogenesis was simulated.

Spatial information in the hippocampus is represented by PFs; thus, for each GC, each PF in each environment was analyzed. Our results demonstrated that the number of PFs in the place maps of the encoding cells was slightly higher than the number of PFs in the network without neurogenesis; these values are similar to those that have been obtained in previous experiments in rats ${ }^{7,9,16}$. Another interesting issue to be analyzed is the area of the PFs. In this case, both of the networks exhibited the same statistical distribution of PF areas, and neither set of PFs exhibited a normal area distribution. Therefore, calculating the mean and the standard deviation would not be representative of their characteristics. Other authors have performed this type of relative area analysis and have obtained similar values ${ }^{7,9}$. All of these results suggest that architectural changes in the neural network do not alter how the place maps are generated in the GCs of the DG.

As described in the Introduction, rate remapping is a process through which spatial representation, as defined by PFs, changes firing frequencies if the sensory stimuli are slightly altered ${ }^{10}$. Because PFs can grow or decrease in size in a given place map ${ }^{7,10,13}$, we considered the absolute value of these changes to be the primary factor to be quantified; we referred to this quantity as the coefficient of rate remapping. When PFs are modified by a novel environment via the modification of synaptic weights, the original place maps will last for only a short time in the memory and will therefore be forgotten ${ }^{13,14}$. We observed that in a neurogenic DG, the encoding cells exhibited a lower mean rate remapping coefficient than that exhibited by the encoding cells of a non-neurogenic DG. To confirm the existence of differences between these two DG types, we evaluated the effect size by calculating Hedge's g (unbiased); this standard measure of effect size revealed a value of 0.754 , which indicates a moderate-to-strong difference. These results confirm the hypothesis that neurogenesis is an effective mechanism that prevents the modification of place maps.

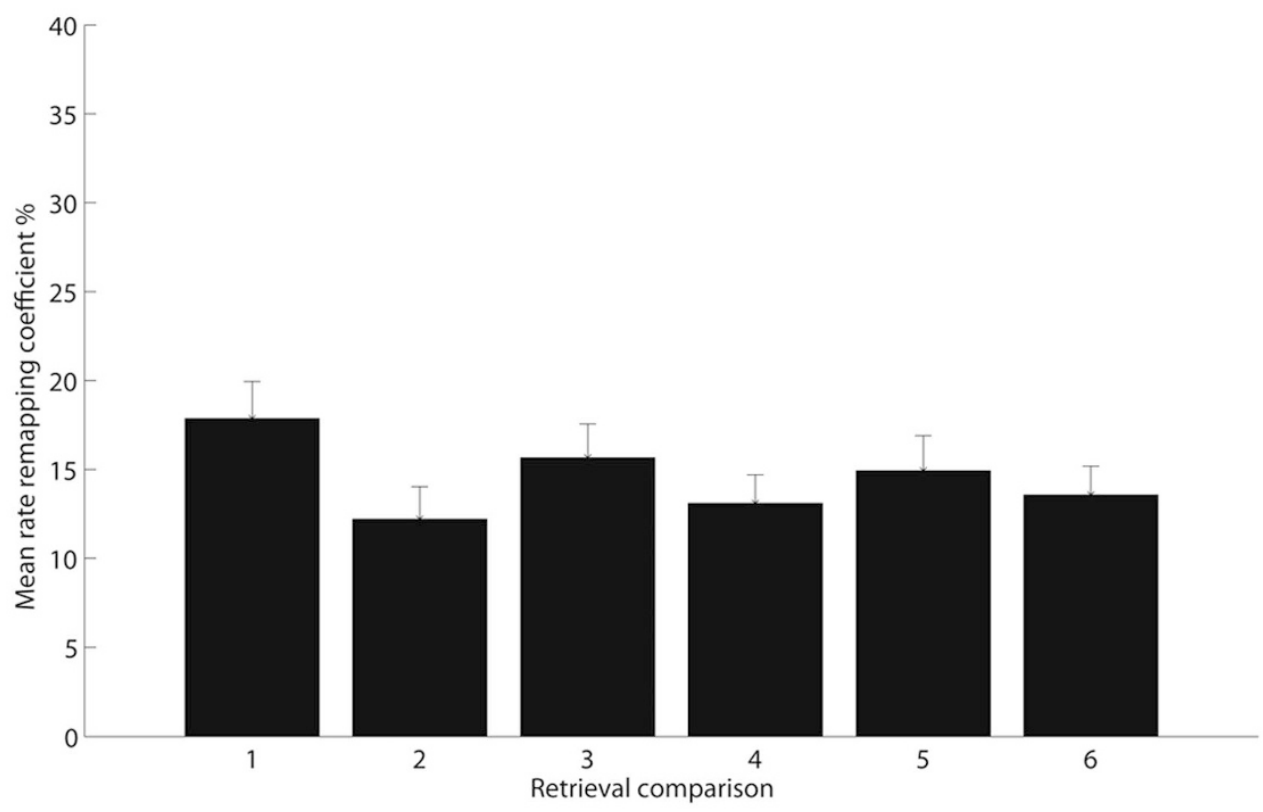

Figure 3 Mean rate remapping coefficient in a network with neurogenesis. The mean rate remapping coefficient of the retrieval comparisons (mean \pm s.e.m.). The mean rate remapping of the columns was $14.25 \% \pm 10.58$ (mean \pm s.d. pool, $N=6$ ). Thus, in a network where neurogenesis occurs, the magnitude of change in the PFs of each cell showed no statistically significant difference. 


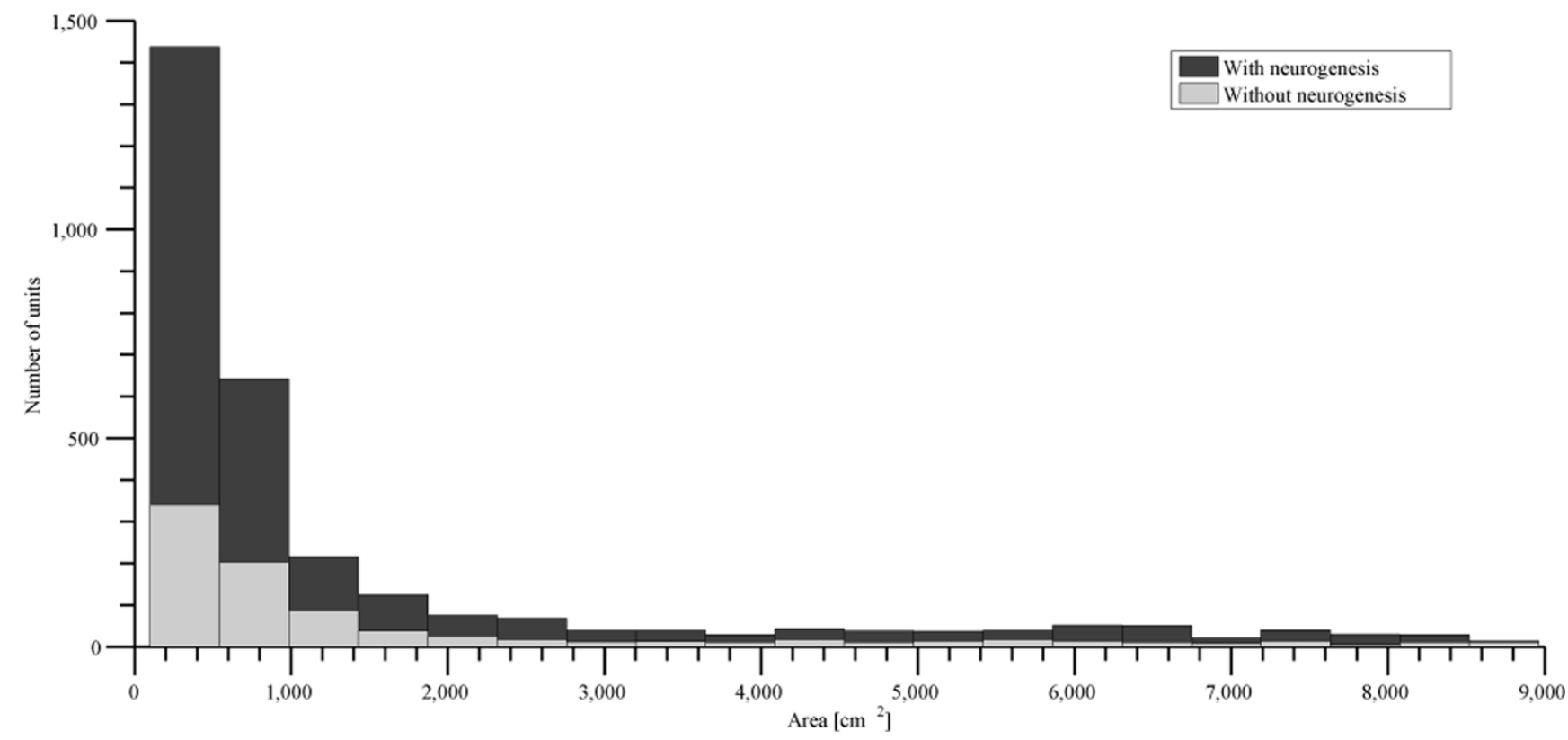

Figure $4 \mid$ Histogram of the areas of the place fields of the encoding neurons. The light-gray bars represent the areas of the place fields of a DG without neurogenesis, and the gray bars represent the areas of the place fields of a DG with neurogenesis. None of these distributions were normally distributed.

Aimone demonstrated that familiar environments attain dimensional independence; this hypothesis was similar to the hypotheses that was proposed by previous authors ${ }^{17,18}$. These authors proposed that neurogenesis could prevent the collapse of previously encoded memories. Alternatively, Aimone noted that the cessation of neurogenesis results in a subtler deficit; novel environments would continue to be encoded using a combination of previous environments. Our results indicate that the cessation of neurogenesis modifies the representation of the spatial information that is encoded in the GC due to a higher degree of rate remapping on the PF of the encoding cells, which thus could produces interference between memories. These results create a theoretical framework for future animal research into the effects of neurogenesis on place maps. These studies will attempt to validate the hypothesis that is presented here. In the future, it will be necessary to record the spatial map that is represented with and without neurogenesis on the encoding cells using experiments in which the animal experiences different environmental types (such as open fields). This experiment will require a full or partial recording of the activity of the entire GC population of the DG. This recording will be achieved by introducing additional electrodes into the hippocampus.

The objective this study was to examine the effects of neurogenesis on the DG. We demonstrated that although architectural alterations occur in the network due to neurogenesis, the manner in which the place maps are generated does not change. However, we observed that neurogenesis diminishes the remapping that is observed in the PFs of the encoding GC and, in this manner, diminishes the interference that is produced by novel environments.

\section{Methods}

Neural network architecture. The model consisted of six layers of different cell types. The layers consisted of the medial entorhinal cortex cells (mECs), the lateral entorhinal cortex cells (lECs), the granule cells layer (GCs), the basket cells layer (BCs), the mossy cells layer (MCs) and the hilar cells layer (HIs). The GC layer was the only layer to which new cells could be added over time through neurogenesis. Eight hundred adult GCs were initially created to produce a fully connected DG that could interact with the model's inputs (see Fig. 5). This is an abbreviated description of the computational methods that were used in the present study. For a more complete description of the model, please see the supplementary description of the model.

Experimental procedure. As a base model, we utilized the model that was proposed by Aimone et al. $2009^{2}$. This base model was adapted for our study and included a new type of learning-testing protocol to examine the effect of neurogenesis on the spatial encoding of DG GCs. The experiment was divided into two phases as follows:

- The training phase consisted of eight different environments that the model was required to learn. Each environment was characterized by a particular configuration for each $\mathrm{mEC}$ cell and different sensory inputs that were represented by the activity of the lEC.

- The testing phase was performed following the learning of a novel environment. At this stage, all of the learned environments were tested using a more thorough analysis to detect changes in the coding cells for each environment (see Fig. 6).

Each time the model was submitted to an environment was referred to as an event. Each event consisted of a 60 -s interval that was divided into 25 -ms steps, during which a virtual rat could explore the environment. In the training phase, the rat spent 40 days in each environment, during which time the model was exposed to the environment 10 times a day for a total of 400 events per environment. In the testing phase, for each learned environment, the model was exposed to a total of 200 events. During this phase, the activity of the cells was registered for later analysis. A flowchart of the experiment is illustrated in Supplementary Fig. S1.

Each event involved a new path for the rat in each environment, ensuring that the entire environment was explored.

Encoding cell detection. To identify the cells that encode each environment, the firing rate of each cell in the layer was calculated to distinguish between the cells with low firing rates and those with high firing rates. To filter out cells that are uninvolved in a given environment, a threshold of $1 \mathrm{~Hz}$ was used (see Supplementary Fig. 10 and Supplementary Fig. 11).

Acquisition of the firing rate maps. To examine the spatial coding, a $1 \mathrm{~m}^{2}$ open field, which was considered to be an environment, was partitioned into squares of $1 \mathrm{~cm}^{2}$, in which cellular activity was analyzed.

After the spikes were spatially distributed, a map of the firing rate was generated based on how many times the rat was situated at each position, representing the firing rate of each position in the environment. The firing rate maps were subjected to a Gaussian filter to soften the frequency map, and the maps were reassembled into longer registers, producing firing rate maps as shown in Supplementary Fig. S7.

Detection of the place fields. As the first step in the analysis of the PFs of the encoding cells, we detected the PFs using our proposed algorithm. In this model, the firing rate maps were subjected to several analytical steps to detect the regions of interest of the firing rate maps. Theoretically, the 3-D shape that best approximates the shape of a PF is a 3-D Gaussian bell. If a horizontal section of a Gaussian bell is taken at its base, an ellipse in the 2-D plane will be obtained. This method was used to obtain a 2-D representation of the place maps. The regions that fell within the approximate circumference of this ellipse were considered to be regions of interest. These regions were filtered by their shape and size to obtain a final sample of PFs (see Supplementary Fig. S8). For a more complete description of the algorithm, please see the supplementary information. 


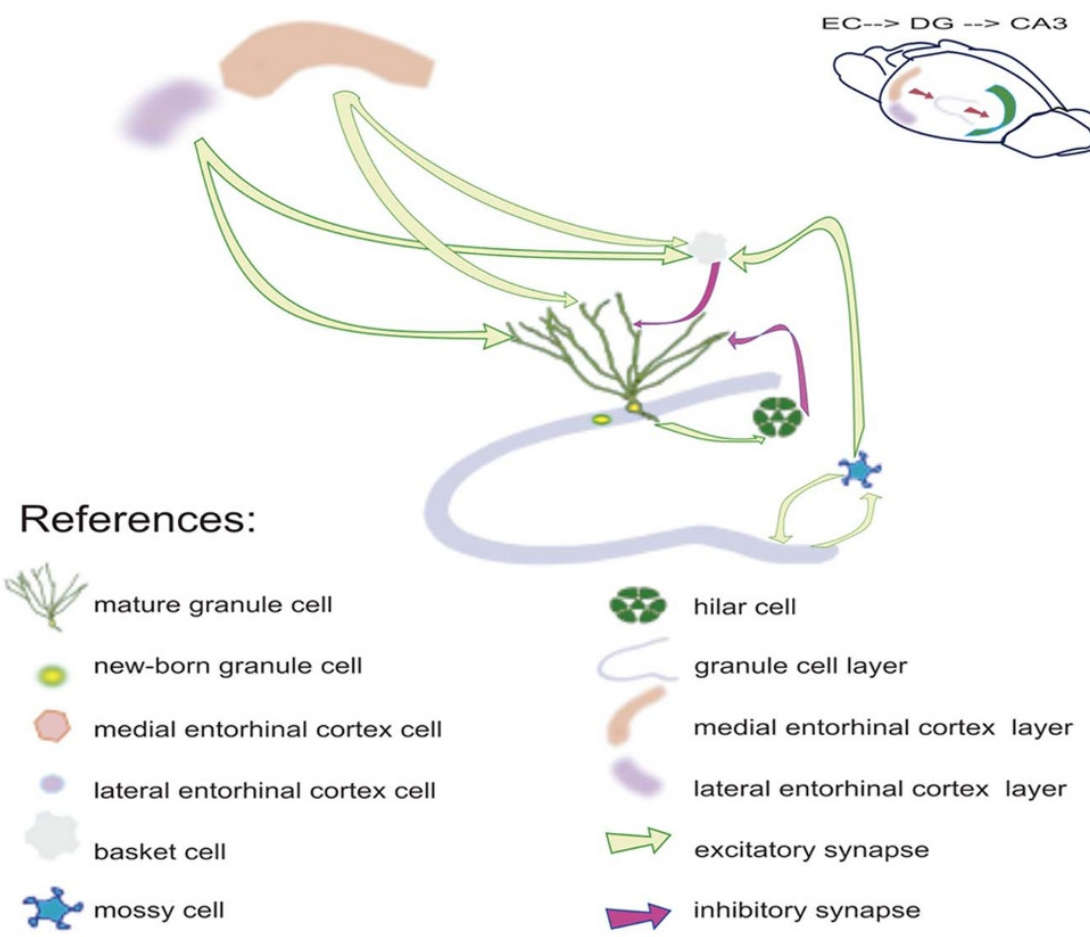

Figure $5 \mid$ Scheme of the neural network used. The magenta lines indicate GABAergic synapses, and the green lines indicate glutamatergic synapses. The orange and violet neurons constitute the inputs of the network (the mECs and lECs) and carry the spatial information (mECs) and sensory properties of the environment (IECs). It can be observed that the GC layer can grow over time via neurogenesis.

\section{Environments}
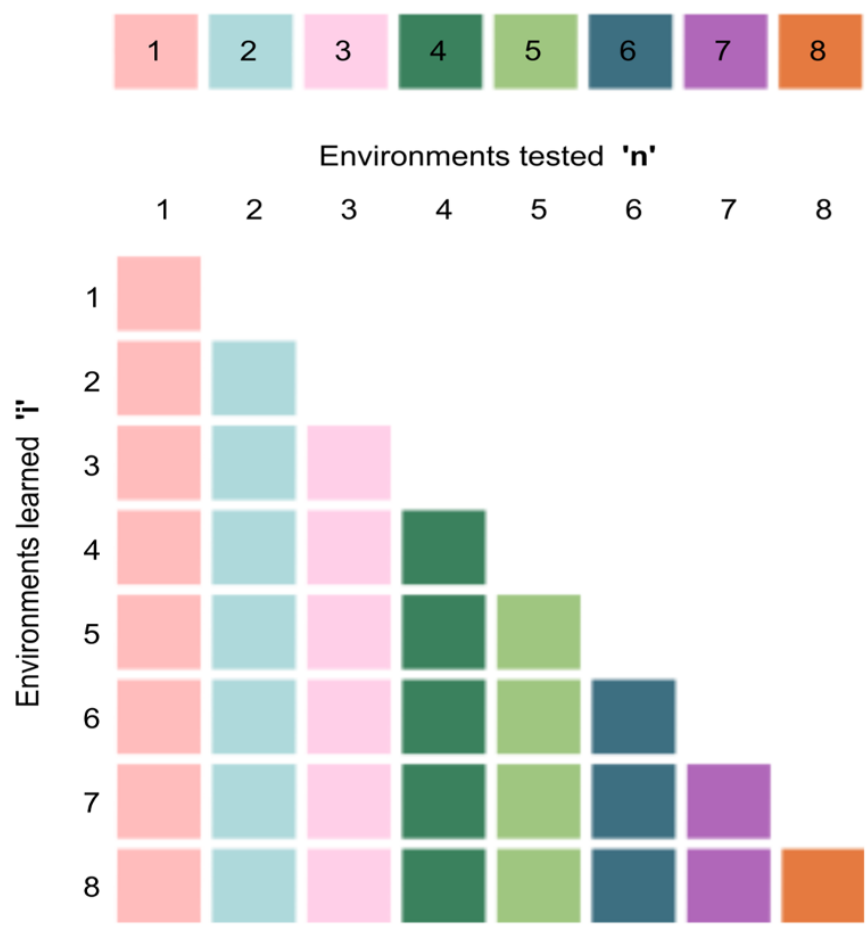

Figure 6 Schematic diagram of the testing phase. In this figure, $\mathbf{i}$ represents the learned environment, and $\mathbf{n}$ represents the environment tested. For each novel environment that was learned, each of the previously learned environments was tested. For each of the environments, a new representation was generated for each novel environment learned. Therefore, when the eight environments were learned, eight different representations of environment one were recorded for analysis.
Place field analysis. As explained in the description of the experimental procedures, all of the previous environments were tested in each novel learned environment, such that we ultimately obtained a series of registers for each of the environments (see Fig. 6). For each retrieval, the PFs were re-determined and labeled with the coefficient d, representing the maximum firing rate of the PF. Fig. 1 depicts how the PF was labeled with the coefficient $\mathbf{d}$ with each new memory for an environment.

The coefficient $\boldsymbol{\rho}$ is the coefficient of the rate remapping, and it is a percentage measure of the degree to which the coefficient $\mathbf{d}$ was modified with the new memory. $\rho$ was calculated using equation (1.1).

$$
\rho_{n, j, i}=\frac{\left(d_{n}, j, i-d_{n-1, j, i}\right)}{d_{n-1, j, i}} \times 100
$$

$\rho_{n, j, i}$ : the coefficient of rate remapping of the place field $\mathbf{j}$ on retrieval $\mathbf{n}$ of environment i.

$d_{n, j, i}$ : the maximum firing rate of the place field $\mathbf{j}$ on retrieval $\mathbf{n}$ of environment $\mathbf{i}$.

The coefficient $\mathbf{d}$ of each of the PFs was determined for each new retrieval of an environment. A new coefficient $\rho$ could therefore be calculated. This method produces a coefficient matrix in which the columns represent the retrieval comparison $\mathbf{n}$ of the environment, the rows represent the different PFs $\mathbf{m}$ of the encoding cells and the sub-index $i$ represents the environment under analysis. This matrix is indicated in equation (1.2).

$$
\left[\begin{array}{ccc}
\rho_{1,1, i} & \cdots & \rho_{1, n, i} \\
\vdots & \ddots & \vdots \\
\rho_{m, 1, i} & \cdots & \rho_{m, n, i}
\end{array}\right]
$$

To examine the changes that occurred following the formation of new memories, we propose to use the mean of the absolute values of the columns of equation (1.2), which represent the average change for all of the coding cells. This mean is described in equation (1.3).

$$
\left[\operatorname{mean}\left(\operatorname{abs}\left(\rho_{1: m, 1, i}\right)\right) \quad \cdots \quad \operatorname{mean}\left(\operatorname{abs}\left(\rho_{1: m, n: i}\right)\right)\right]
$$

$m$ : is the total number of PFs that form matrix (1.2) (number of rows). $n$ : is the column of matrix (1.2) that represents the retrieval comparison. $i$ : is the environment analyzed.

1. Barinaga, M. Newborn Neurons Search for Meaning. Science 299, 32-34 (2003).

2. Aimone, J. B., Wiles, J. \& Gage, F. H. Computational Influence of Adult Neurogenesis on Memory Encoding. Neuron 61, 187-202 (2009).

3. O'Keefe, J. \& Dostrovsky, J. The hippocampus as a spatial map. Preliminary evidence from unit activity in the freely-moving rat. Brain Res. 34, 171-175 (1971). 
4. O'Keefe, J. Place units in the hippocampus of the freely moving rat. Exp. Neurol. 51, 78-109 (1976).

5. McNaughton, B. L., Battaglia, F. P., Jensen, O., Moser, E. I. \& Moser, M.-B. Path integration and the neural basis of the 'cognitive map'. Nat Rev Neurosci 7 , 663-678 (2006).

6. Colgin, L. L., Moser, E. I. \& Moser, M.-B. Understanding memory through hippocampal remapping. Trends Neurosci. 31, 469-477 (2008).

7. Leutgeb, J. K., Leutgeb, S., Moser, M.-B. \& Moser, E. I. Pattern Separation in the Dentate Gyrus and CA3 of the Hippocampus. Science 315, 961-966 (2007).

8. Molter, C. \& Yamaguchi, Y. Entorhinal theta phase precession sculpts dentate gyrus place fields. Hippocampus 18, 919-930 (2008).

9. de Almeida, L., Idiart, M. \& Lisman, J. E. The Input-Output Transformation of the Hippocampal Granule Cells: From Grid Cells to Place Fields. J. Neurosci. 29, 7504-7512 (2009).

10. Rennó-Costa, C., Lisman, J. E. \& Verschure, P. F. M. J. The Mechanism of Rate Remapping in the Dentate Gyrus. Neuron 68, 1051-1058 (2010).

11. de Almeida, L., Idiart, M. \& Lisman, J. E. The single place fields of CA3 cells: A two-stage transformation from grid cells. Hippocampus 22, 200-208 (2012).

12. Muller, R. \& Kubie, J. The effects of changes in the environment on the spatial firing of hippocampal complex-spike cells. J. Neurosci. 7, 1951-1968 (1987).

13. Leutgeb, S. et al. Independent Codes for Spatial and Episodic Memory in Hippocampal Neuronal Ensembles. Science 309, 619-623 (2005).

14. Treves, A., Tashiro, A., Witter, M. P. \& Moser, E. I. What is the mammalian dentate gyrus good for? Neurosci. 154, 1155-1172 (2008).

15. Aimone, J. B. \& Gage, F. H. Modeling new neuron function: a history of using computational neuroscience to study adult neurogenesis. Eur. J. Neurosci. 33, 1160-1169 (2011).

16. Park, E., Dvorak, D. \& Fenton, A. A. Ensemble Place Codes in Hippocampus: CA1, CA3, and Dentate Gyrus Place Cells Have Multiple Place Fields in Large Environments. PLoS ONE 6(7) (2011).

17. Appleby, P. A. \& Wiskott, L. Additive neurogenesis as a strategy for avoiding interference in a sparsely-coding dentate gyrus. Network. 20, 137-161 (2009).
18. Wiskott, L., Rasch, M. J. \& Kempermann, G. A functional hypothesis for adult hippocampal neurogenesis: Avoidance of catastrophic interference in the dentate gyrus. Hippocampus 16, 329-343 (2006).

\section{Acknowledgements}

We are grateful to Maria Belen Viano, who helped edit the English in the manuscript. We also wish to thank Lucas Damian Costa for his help with the statistics and Guido Benjamin Guzman for his support in the revision of the manuscript. This study was supported by a grant from the Fundación para el Desarrollo de las Ciencias Básicas (FUCIBA) (the Foundation for the Development of Basic Sciences).

\section{Author contribution}

P.F.A. developed the project ideas. J.I.C. and N.H.Q. worked together to develop the neural network and to perform the data analysis. P.F.A. was the supervisor who was responsible for the project planning and co-wrote the manuscript with J.I.C. and N.H.Q. V.I.W edited the manuscript in collaboration with P.F.A.

\section{Additional information}

Supplementary information accompanies this paper at http://www.nature.com/ scientificreports

Competing financial interest: The authors declare no competing financial interests.

License: This work is licensed under a Creative Commons

Attribution-NonCommercial-NoDerivative Works 3.0 Unported License. To view a copy of this license, visit http://creativecommons.org/licenses/by-nc-nd/3.0/

How to cite this article: Cuneo, J.I., Quiroz, N.H., Weisz, V.I. \& Argibay, P.F. The computational influence of neurogenesis in the processing of spatial information in the dentate gyrus. Sci. Rep. 2, 735; DOI:10.1038/srep00735 (2012). 